\title{
Solar off-limb line widths with SUMER: revised value of the non-thermal velocity and new results
}

\author{
L. Dolla ${ }^{1}$ and J. Solomon ${ }^{2,3}$ \\ ${ }^{1}$ Solar-Terrestrial Center of Excellence-SIDC, Royal Observatory of Belgium, Avenue Circulaire 3, 1180 Brussels, Belgium \\ ${ }^{2}$ Institut d'Astrophysique Spatiale, UMR8617, Université Paris-Sud, 91405 Orsay, France \\ ${ }^{3}$ CNRS, Orsay 91405, France
}

Received: 22 May 2009 - Revised: 12 August 2009 - Accepted: 4 September 2009 - Published: 25 September 2009

\begin{abstract}
Alfvén waves and ion-cyclotron absorption of high-frequency waves are frequently brought into models devoted to coronal heating and fast solar-wind acceleration. Signatures of ion-cyclotron resonance have already been observed in situ in the solar wind and in the upper corona. In the lower corona, one can use the line profiles to infer the ion temperatures. But the value of the so-called "non-thermal" (or "unresolved") velocity, potentially related to the amplitude of Alfvén waves propagating in the corona, is critical in firmly identifying ion-cyclotron preferential heating. In a previous paper, we proposed a method to constrain both the Alfvén wave amplitude and the preferential heating, above a polar coronal hole observed with the SUMER/SOHO spectrometer. Taking into account the effect of instrumental stray light before analysing the line profiles, we ruled out any direct evidence of damping of the Alfvén waves and showed that ions with the lowest charge-to-mass ratios were preferentially heated. We re-analyse these data here to correct the derived non-thermal velocity, and we discuss the consequences on the main results. We also include a measure of the FeviII $1442.56 \AA$ line width (second order), thus extending the charge-to-mass ratio domain towards ions more likely to experience cyclotron resonance.
\end{abstract}

Keywords. Solar physics, astrophysics, and astronomy (Corona and transition region; Ultraviolet emissions)

\section{Introduction}

Several models have been proposed to heat the corona and accelerate the fast solar wind. Some of them include Alfvén waves and/or ion-cyclotron resonance as a dissipation process of the high frequencies waves (e.g., Tu and Marsch,

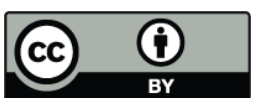

Correspondence to: L. Dolla

(dolla@sidc.be)
1997; Hu et al., 2000; Vocks, 2002; Bourouaine et al., 2008b; Landi and Cranmer, 2009; Isenberg and Vasquez, 2009). In this respect, coronal holes are of particular interest as the source of the fast solar wind. Alfvén waves have long been observed in situ in the solar wind (see references in Schwenn and Marsch, 1991), while direct observations near the Sun are still not ascertained. Tomczyk et al. (2007) observed intensity and velocity fluctuations propagating along the field lines that they interpret as Alfvén waves, but Van Doorsselaere et al. (2008b,a) interpret them as fast kink mode. Besides, these observations are essentially made in close field lines and not coronal holes. Ioncyclotron resonance can cause anisotropies of temperatures $\left(T_{\perp} / T_{\|}\right)$and preferential heating of heavy ions. This latter is due to the charge-to-mass ratio $(q / \mathrm{m})$ dependance of the ion-cyclotron frequency $\omega_{c}=q B / m$, where $B$ is the local magnetic field. Such signatures have been observed in situ in the solar wind (e.g. von Steiger et al., 1995, with the Ulysses spacecraft), and in the corona with remote-sensing methods and different instruments (with the Solar Ultraviolet Measurements of Emitted Radiation (SUMER) spectrometer onboard the SOlar and Heliographic Observatory (SOHO) spacecraft: Tu et al. (1998); the Large Angle and Spectrometric COronograph (LASCO) onboard SOHO: Mierla et al. (2008); the UltraViolet Coronagraph Spectrometer (UVCS) onboard SOHO: Cranmer et al., 2008). One has to note, though, that anisotropies of temperature could also be produced by non-resonant Alfvén waves (Bourouaine et al., 2008a).

Most of the time, the remote-sensing techniques used to study ion temperatures rely on the analysis of line widths. When the plasma is optically thin for the line wavelength $\lambda$, the half-width $\sigma$ at $1 / \sqrt{e}$ of the line profile is given by the following formula (e.g. Dere and Mason, 1993):

$\sigma^{2}=\frac{\lambda^{2}}{2 c^{2}}\left(\frac{2 k T}{m}+\xi^{2}\right)$

Published by Copernicus Publications on behalf of the European Geosciences Union. 
where $T$ is the ion temperature, $m$ the ion mass, $c$ the speed of light and $k$ the Boltzmann constant. The "unresolved" velocity, or "non-thermal" velocity $\xi$ is proportional to the mean velocity fluctuations $\left\langle\delta v^{2}\right\rangle^{1 / 2}$, that is, the Doppler variations of the line centre after integration over the observed solid angle (line-of-sight (LOS) and resolution scale) and the exposure time. The coefficient of proportionality, relatively close to 1 , depends on the degrees of freedom of the fluid motion (e.g. Tu et al., 1998).

Things are then complicated by the presence of a double information in the same observable. That is, the result of any heating process (the temperature) and the possible energy source, as the Alfvén wave amplitude is often said to be the origin of the non-thermal velocity. The usual method to tackle this problem is to make a strong assumption on one of the quantities, and then to deduce the other. For instance, the most constraining hypothesis is to assume that the temperature is the temperature of maximum ionisation of the ion species, which of course preclude any conclusion about the preferential heating. On the contrary, Tu et al. (1998) do not attempt to measure the non-thermal velocity and analysed the effect of varying $\xi$ between 0 and the maximum possible value (the largest one that implies no negative temperature of the heaviest ions species in the data set). They then discuss the ion temperatures and thermal speeds as a function of the charge-to-mass ratio, in the resulting ranges of these quantities. But it appears that the derived temperatures of heavy ions like the iron ones are very sensitive to the value of $\xi$. Unfortunately, these heavy ions are also the ones having the lowest $q / m$, that is, that are more likely to be heated by ion-cyclotron resonance. Consequently, better constraining $\xi$ would greatly improve the analysis of the preferential heating. And a better constrain on $\xi$ is anyway valuable for models that use Alfvén waves.

The study of line widths and their interpretation is an old issue. As pointed out by Wilhelm et al. (2005), their variation with height largely depends on the solar conditions. In the low corona, there have been studies of their variation with height in coronal holes (Hassler et al., 1990; Banerjee et al., 1998) or above equatorial quiescent region (Harrison et al., 2002; Wilhelm et al., 2005). These are observations of EUV lines, but one can also observe visible or near-infrared lines. A more detailed review can be found in Dolla and Solomon (2008).

The purpose of the present study is to determine what we can say and what we cannot, simply by judging from the data and using relatively "soft" hypotheses. In particular, we try to constrain the presence or not of preferential heating in what proves to be the least favorable case, i.e. the presence of undamped Alfven waves. A method has been proposed and tested in Dolla and Solomon (2008), hereafter Paper I. We re-analyse here the same data to correct one intermediate formula (the computation of $v^{2}$ from the line widths data, see below), and to make use of newer atomic data for the density diagnostic: the CHIANTI database (Dere et al., 1997; Landi et al., 2006) instead of the line ratio computed in Doschek et al. (1997). We also analyse a recently identified Fe VIII line. This is now the ion with the smallest charge-to-mass ratio $(0.125)$ in our data set.

\section{Observations and data analysis}

The observation, data processing and method to distinguish the effects of temperature and non-thermal velocity are detailed in Paper I. We only remind the main points here.

\subsection{Observations}

Observations were made with the VUV spectrometer SUMER/SOHO (Wilhelm et al., 1995) on May, 30th 2002, during Medoc Campaign \#9. The SUMER slit was positionned above the North polar coronal hole $(X=0, Y=1150)$, in the North-South direction. The apparent solar diameter was $956.5^{\prime \prime}$. Several spectral ranges were observed during at least a one-hour exposure each. An optimal instrumental width for profile analysis was achieved by using both the $1^{\prime \prime} \times 300^{\prime \prime}$ slit and detector A.

Raw data were corrected using the standard SUMER procedures provided by Solar Software (flatfield and geometric distortion of the detector). For better statistics, we integrated the spectral profiles over several spatial pixels (running sum over 40 spatial pixels, with a sliding step of 1 pixel). The present re-analysis focus on the main lines that can be observed up to high altitude above the surface, and that are listed in Table 1. As compared to Paper I, we added the newly identified $1442.56 \AA \mathrm{Fe}$ VIII line (Ekberg and Feldman, 2003), available in the data set between the 1440 and the $1445 \AA \mathrm{Si}$ VIII lines and observed in second order. We use here the wavelength measured in Feldman et al. (1997), like for the other lines. This line is very faint, and the fitting procedure fails above $100^{\prime \prime}$. It is nevertheless of great interest because of the relatively small charge-to-mass ratio. Hence, this is now the ion species that is the most likely to be heated by ion cyclotron resonance.

\subsection{Removing the instrumental stray light}

As SUMER is not a coronagraph, off-limb observations are contaminated by instrumental scattered light coming from the disc. This stray light contribution is revealed by persistent cold lines at high altitude, whose relative intensity as compared to hotter lines even becomes more important as the altitude increases. But in fact, the stray light spectrum is composed of both cold and hot lines. It is indeed a typical on-disc spectrum, that is simply added, with a contribution factor that depends on the altitude, latitude and time of observation, to the "real" off-limb spectrum. Then, the coronal lines observed above the limb can be blended with lines emitted by another or by the same ion species, seen as a "ghost" of the on-disc spectrum. In both cases, the contaminating 
Table 1. Observed coronal lines.

\begin{tabular}{lcccc}
\hline ion & $\lambda(\AA)^{\mathrm{a}}$ & $\log T_{\max }{ }^{\mathrm{b}}$ & atomic mass & $q / m$ \\
\hline Fe VIII & $1442.56^{*}$ & 5.80 & 55.85 & 0.13 \\
Fe X & 1028.02 & 6.08 & 55.85 & 0.16 \\
Fe X & 1463.50 & 6.08 & 55.85 & 0.16 \\
Fe XII & 1349.36 & 6.22 & 55.85 & 0.20 \\
Fe XII & 1242.00 & 6.22 & 55.85 & 0.20 \\
Ca X & $1148.00^{*}$ & 5.87 & 40.08 & 0.22 \\
Si VIII & 1445.75 & 5.92 & 28.08 & 0.25 \\
S X & 1196.20 & 6.14 & 32.06 & 0.28 \\
O VI & 1031.93 & 5.58 & 16.00 & 0.31 \\
O VI & 1037.60 & 5.58 & 16.00 & 0.31 \\
Mg IX & $1412.11^{*}$ & 5.99 & 24.31 & 0.33 \\
Na IX & $1363.45^{*}$ & 5.92 & 22.99 & 0.35 \\
Si XI & $1161.82^{*}$ & 6.20 & 28.08 & 0.36 \\
Mg X & $1249.88^{*}$ & 6.04 & 24.31 & 0.37 \\
\hline
\end{tabular}

a Wavelengths in ångstroems are taken from Feldman et al. (1997). Some lines are observed in second order and marked with an*.

b $T_{\max }$ is the temperature of maximum ion abundance.

stray light lines are usually narrower than the coronal ones (see more details in Paper I).

To remove the stray light contribution, we have presented a method in Paper I and related papers. A reference spectrum is taken with SUMER at a very high altitude (pure stray light), which directly provides the spectral profile and the relative intensities of the different lines. Then the absolute intensity of the predicted stray light spectrum is scaled at any altitude by using on the observed coronal spectrum either the intensity of a line identified as stray light (with no blending), or the continuum. Finally, the "real" coronal spectrum is retrieved by subtracting point by point in wavelength the predicted stray light spectrum from the observed one. Examples are given in Dolla et al. (2003, 2004); Dolla and Solomon (2008). Note that no reference spectrum was taken for some spectral ranges, as the stray light contamination of the lines of interest was initially considered negligible. This is in particular the case for the Fe vIII line. According to Curdt et al. (2001), there is a neighbouring Fe II line at $1442.75 \AA$, which may contribute as stray light in off-limb spectra. At altitudes above $70^{\prime \prime}$, a small bump is visible in the red wing of the profile, and above $100^{\prime \prime}$, two components are clearly visible. Further work is therefore needed to quantify this blending and its effects.

The line width $\sigma$ is finally determined by making gaussian fits. The known instrumental width is removed before doing the subsequent analysis.

\subsection{Distinguishing temperature and non-thermal velocity}

To verify if there is both preferential heating and Alfvén waves at the observed locations, we have to suppose that part of the line widths was due to the effect of the (low frequency) Alfvén wave amplitude (the term with $\xi$ in Eq. 1). A reasonable assumption is then to say that the value of $\xi$ is the same for every ion species observed at a given location. Indeed, the reason why the wave amplitude should broaden the line width is that it corresponds to a fluid motion of the whole plasma, so that every ion species from protons to iron ions "see" the same velocity. This is true only if the drift velocity of one species as compared to the proton one is small (Hollweg and Isenberg, 2002).

The next assumption is to consider that these Alfvén waves stay undamped within the observed altitude range. In fact, this hypothesis enables to set a maximum value for $\xi$, and hence, a minimum boundary on the heating. Without damping, the energy flux of the Alfvén waves is conserved, and follow the relation (e.g. Moran, 2001):

$\frac{\xi(r)}{\xi\left(r_{0}\right)}=\left(\frac{\rho(r)}{\rho\left(r_{0}\right)}\right)^{-1 / 4}$

where $r$ is the distance to the Sun centre, $\rho(r)$ is the density, and $r_{0}$ is the reference location for setting the constant of the power law. The density can be determined by measuring the intensity ratio of the $1445 / 1440 \AA \mathrm{Si}$ VIII lines and using the CHIANTI database. But one has to constrain the constant $\xi\left(r_{0}\right)$. An additional assumption is added at this point: the ion-cyclotron heating should be negligible for ion species having the highest $q / m$, because of the preferential heating of the species having the lowest $q / m$. This is supported by simulations of ion-cyclotron resonance including several ions species, that show that the species with the lowest $q / m$ always absorb most of the wave energy and leave nothing to appreciably heat the other species (e.g. Vocks and Marsch, 2002). Then, the line width of $\mathrm{Mg} \times$ can be used as an additional constrain, by supposing that its variation between $r_{0}$ and a given $r$ is solely due to a variation of $\xi$. In other words, the variation in temperature $\Delta T(r)=T(r)-T\left(r_{0}\right)$ is neglected. This can be written as:

$\Delta\left(v^{2}(r)\right)=v^{2}(r)-v^{2}\left(r_{0}\right)=\xi^{2}(r)-\xi^{2}\left(r_{0}\right)$

with the quantity:

$v^{2}=\frac{2 c^{2} \sigma^{2}}{\lambda^{2}}=\frac{2 k T}{m}+\xi^{2}$.

From Eqs. (2) and (3), we get:

$\xi\left(r_{0}\right)=\left[\frac{\Delta\left(v^{2}(r)\right)}{\left(\frac{\rho(r)}{\rho\left(r_{0}\right)}\right)^{-1 / 2}-1}\right]^{1 / 2}$. 


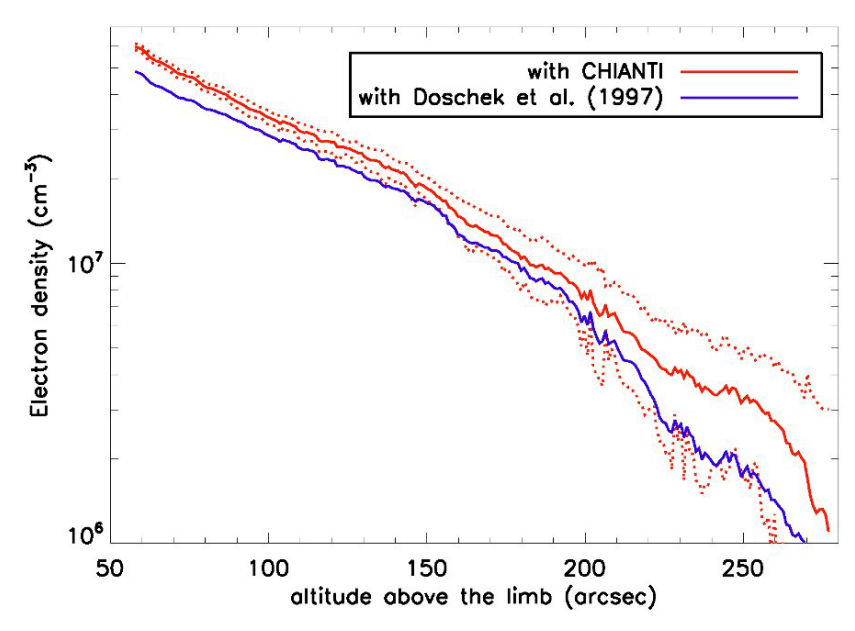

Fig. 1. Electron density with increasing altitude above the polar coronal hole. In red, the new value by using the line ratio computed in the CHIANTI database. In blue, the value previously used in Paper I by using the line ratio data in Doschek et al. (1997). The dotted lines denote the error bars for the new value.

Thus, $\xi\left(r_{0}\right)$ can be determined for any $r$ where the hypothesis $\Delta T(r)=0$ is true for Mg X and where $\rho(r)$ and $v(r)$ can be measured. As a consequence, $\xi(r)$ can then be determined using Eq. (2) everywhere we have $\rho(r)$, with no other hypothesis than flux conservation. The line width of $\mathrm{Mg} \mathrm{x}$ and the hypothesis on the absence of temperature variation is only necessary to constrain the lower boundary $\xi\left(r_{0}\right)$.

Once we have $\xi(r)$, we can determine the temperature of every ion species for which we measure the line width, just by using Eq. (1).

\section{Results and comparison with our previous ones}

It appeared that the factor 2 in Eq. (4) (before $c^{2} \sigma^{2} / \lambda^{2}$ ) was missing in the analysis software that was used in Paper I. This led to an understimation of $\xi\left(r_{0}\right)$ by a factor of $\sqrt{2}$. The derived temperature, and particularly the difference in temperature between altitudes was therefore overestimated. We present here the corrected values, including also new atomic data for the density diagnostic.

\subsection{Density and non-thermal velocity}

The use of newer line ratio data from CHIANTI (version 5.2.1) instead of those taken in Doschek et al. (1997) led to an increase in the derived density (Fig. 1). At 58", for example, it increased from about $5 \times 10^{7}$ to $6 \times 10^{7} \mathrm{~cm}^{-3}$. Note that below $10^{6} \mathrm{~cm}^{-3}$, the line-ratio is not sensitive to the variation of density. We therefore limit the curves at this value.

Applying Eq. (5) with $r_{0}$ corresponding to the lowest observed altitude of the data set $\left(\approx 60^{\prime \prime}\right)$ and for any altitude

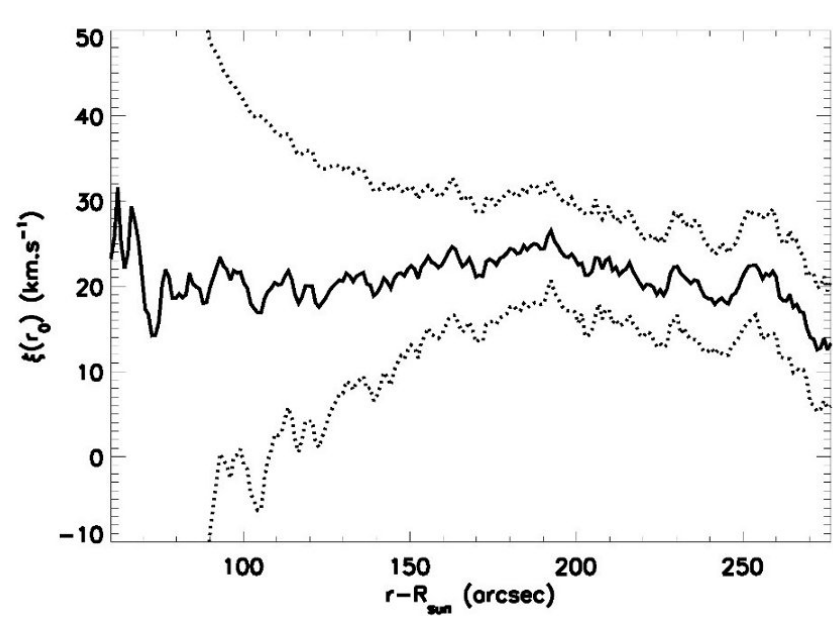

Fig. 2. Value of the non-thermal velocity $\xi\left(r_{0}\right)$ for $r_{0}-R_{\odot} \approx 60^{\prime \prime}$ and $r-R_{\odot}$ varying between $60^{\prime \prime}$ and $270^{\prime \prime}$ (see Eq. 5). The dotted lines denote the theoretical error bars.

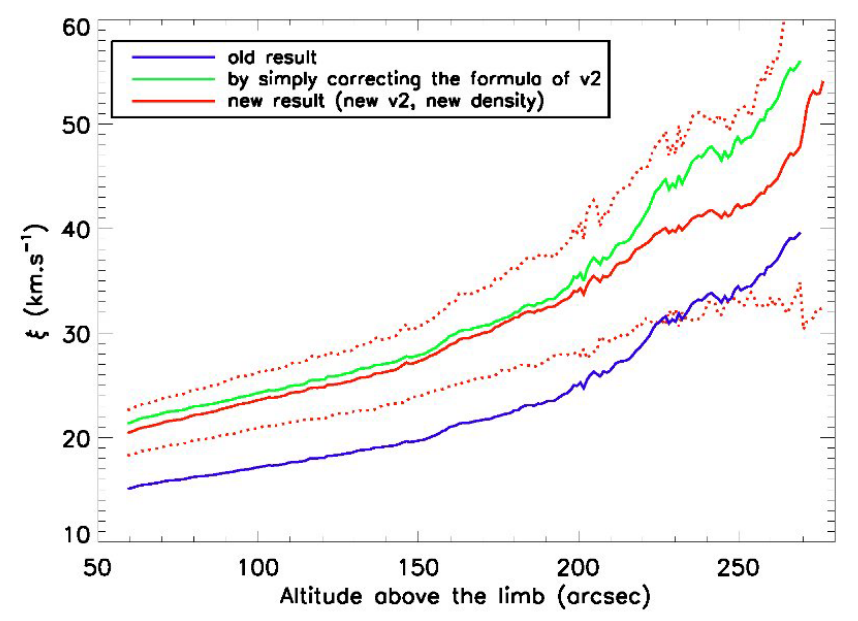

Fig. 3. Non-thermal velocity with increasing altitude above the polar coronal hole. In blue, the value derived in Paper I. In green, when the factor 2 is included in the formula of $v^{2}$ (see text). In red, the new result when both the formula is corrected and the new derived density is taken into account. The red dotted lines denote the error bars for the new value of $\xi$.

$r-R_{\odot}$ between 60 and $270 "$ yields the curve plotted in Fig. 2. Like in Paper I, the computed $\xi\left(r_{0}\right)$ is roughly constant for $r-R_{\odot}$ between 90 and 150, which tends to prove that the hypothesis $\Delta T=0$ is valid on that interval. The averaging is just a way to get a more precise value (see Paper I for details), but one can see that a more restricted interval can be used without large difference in the result. The new value, including the correction of the factor 2 in Eq. (4) and the new density diagnostic, is then $\xi\left(r_{0}\right)=20.5 \pm 2 \mathrm{~km} \mathrm{~s}^{-1}$, instead of $15 \mathrm{~km} \mathrm{~s}^{-1}$. 

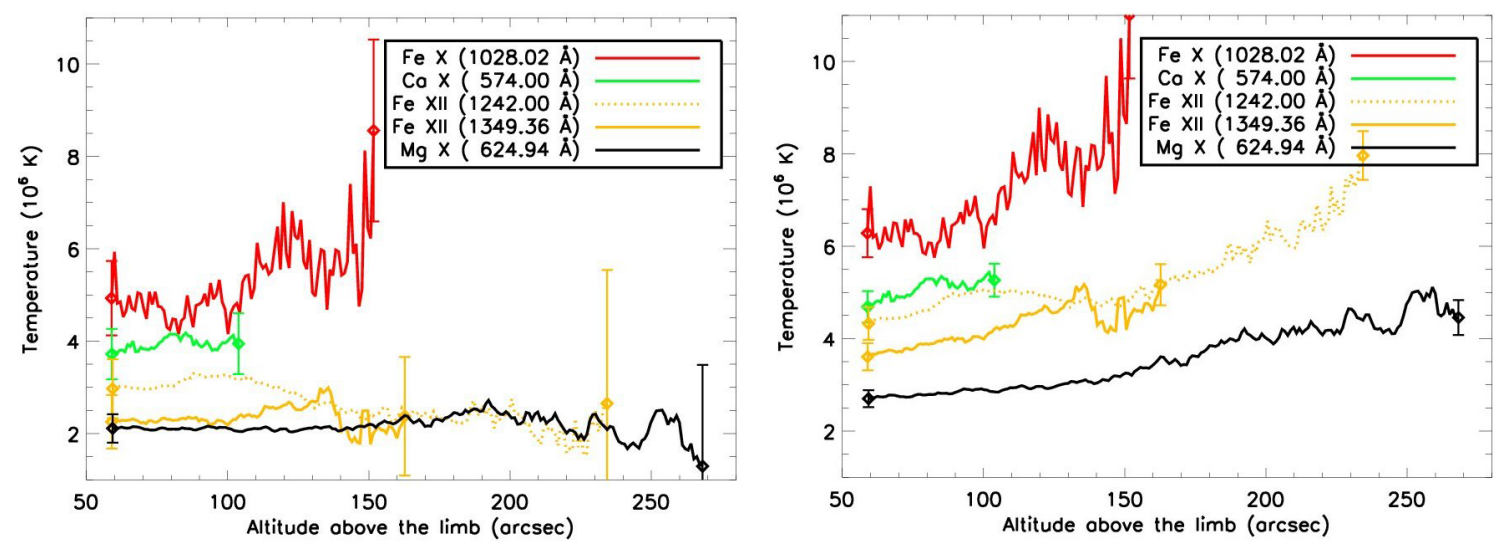

Fig. 4. Temperatures derived from the line widths. Left panel: when the non-thermal velocity is removed. Right panel: when no non-thermal velocity is considered.

With Eq. (2), we can now determine $\xi(r)$ on the interval $\left[60^{\prime \prime}, 270^{\prime \prime}\right]$. Figure 3 show the old result (in blue), the same curve after correction of the factor 2 (in green), and finally the new result once the new density diagnostic is also taken into account (in red). On can notice that the effect of this latter is in fact very small, except above $200 "$.

It is not easy to compare these results to other studies above polar coronal holes. CDS line widths cannot be interpreted in terms of velocity, as the instrumental width is too large. With SUMER, Banerjee et al. (1998) found more than $30 \mathrm{~km} \mathrm{~s}^{-1}$ at height $60^{\prime \prime}$, increasing to $45 \mathrm{~km} \mathrm{~s}^{-1}$ at 200 ", while we find 20 and 35 at these altitudes, respectively. Hassler et al. (1990) give the total line width (thermal and nonthermal) for $\mathrm{Mg} \mathrm{X}$. If we suppose a temperature of $2 \times 10^{6} \mathrm{~K}$, like in our results, a rough estimate can be made for the nonthermal velocity: 36 and $42 \mathrm{~km} \mathrm{~s}^{-1}$, respectively, at 60 and $200^{\prime \prime}$. This goes to 42 and $48 \mathrm{~km} \mathrm{~s}^{-1}$ with the temperature of $1.3 \times 10^{6} \mathrm{~K}$ that they use.

\subsection{Temperature and preferential heating}

Figure 4 shows the temperature as a function of the altitude above the limb for a subset of coronal ion lines. The interval on which they are plotted is different for every ion species, depending on the statistics in the line. Two cases are presented: in the left panel, the non-thermal velocity is removed, while in the right panel the whole width is interpreted in terms of temperature. The first case corresponds to the maximum value of $\xi$ that can be removed (constrained with the density and the gradient of the width of $\mathrm{Mg} \mathrm{X}$ ), and the second case corresponds to the other extreme point of view $(\xi=0)$. As it was already shown in Paper I, any evidence of heating is dramatically reduced when the non-thermal velocity is taken into account. This effect is more important for the heaviest ions (iron), as the thermal contribution in the line width is smaller for them, while the non-thermal contribution is similar for every ion species. This is probably the reason why the hypothesis " $\Delta T=0$ in the lowest altitudes", for $\mathrm{Mg} \mathrm{X}$, works so well, besides its less sensitivity to ioncyclotron preferential heating. Whatever the hypothesis on $\xi$ (first or second panel of Fig. 4), this ion species presents a negligible variation of temperature as a function of the altitude.

With the larger value of $\xi$ that we derived in this work as compared to Paper I, one can no longer see any noticeable heating with the altitude except, maybe, for Fe X. If there is ion-cyclotron preferential heating, Fe $\mathrm{x}$ is the only one that benefits from it above $60^{\prime \prime}$. The width of the Fe VIII line is decreasing softly between 60 " and 100" (not shown). Once we take into account the non-thermal velocity, this implies that the resulting temperature is decreasing with height. This contradicts the interpretation of the increase of the Fe x $1028 \AA$ line as ion-cyclotron heating. But this decreasing width may be due to the stray light blending mentionned above.

Figure 5 shows the ion temperature as a function of the $q / m$ ratio, at $60^{\prime \prime}\left(\approx 1.06 \mathrm{R}_{\odot}\right)$. The non-thermal contribution derived above is removed, so that these values correspond to the lowest possible boundary of the true ion temperatures (without considering $\xi$, temperatures are obviously larger). It appears, first, that all these temperatures are larger than the temperature of maximum ionisation of the corresponding species. This means that one cannot use the usual assumption of temperature of maximum formation to derive the non-thermal velocity. Moreover, these ion temperatures are larger than the electron one, at least by a factor of 2 . The electron temperature is usually measured, indeed, around $10^{6} \mathrm{~K}$ in coronal holes, e.g. Habbal et al. (1993); David et al. (1998); Wilhelm et al. (1998); see in particular the revision of the computation of the electron temperature diagnostic with Mg IX by Del Zanna et al. (2008). Therefore, it appears that some preferential process already heated the ions, as a whole, as compared to the electrons. And this has taken place at lower altitudes than we observed. 


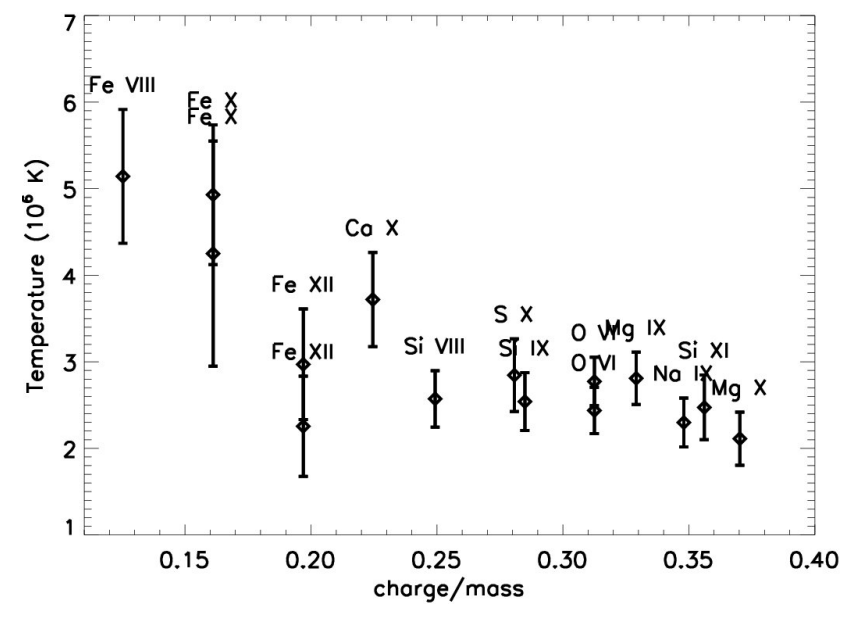

Fig. 5. Ion temperatures at $60^{\prime \prime}$, as a function of the charge-to-mass ratio.

Even with a larger non-thermal velocity than in Paper I, the Fe $x$ species still has a larger temperature than the other ions. We are particularly confident in this result, since a large temperature is obtained with two different lines. The Fe viII temperature confirms that ions with the lowest charge-to-mass ratios are hotter than the other ones.

Note that if the waves are in fact damped then the nonthermal velocity is reduced and the derived temperatures are larger. Especially, the charge-to-mass trend is emphasised (see more details in Paper I). This reinforce the interpretation in terms of ion-cyclotron resonance. Nevertheless, according to Parker (1991), low-frequency Alfvén waves are difficult to damp in the low corona.

We restricted this study to a non-thermal broadening due to the low frequency Alfvén waves. Their effect is quantitatively the same for every ion species and can be simply determined. In case of fast kink mode (dispersive), a relation similar to Eq. (2) has to be derived. One can also consider the case where the resonating ion species have an increased velocity perpendicular to the main magnetic field (Ofman et al., 2005). In this case, one has to distinguish, besides the average amplitude of the low-frequency Alfvén waves and the thermal speed of the ion population, an additional component that is the average fluid velocity of the resonating ion species induced by the resonance. The importance of this effect depends on the spectrum of the circularly polarised waves and requires additional modelling.

\section{Conclusions}

We have re-analysed the off-limb coronal hole data presented in Paper I, improving the method. It enables us to take into account the hypothesis of Alfvén waves that broaden the coronal line profiles, without precluding the study of the in- dividual ion temperatures. This can help to constrain theoretical and numerical models. After correcting a mistake in the analysis software and including newer atomic calculations, the non-thermal velocity have been increased by about $36 \%$ at $60^{\prime \prime}$ (from 15 to $20.5 \mathrm{~km} \mathrm{~s}^{-1}$ ), which reduces, as compared to Paper I, the ion temperatures derived from the line widths.

But most of the conclusions stay unchanged. The nonthermal velocity still remains quite low as compared to the values determined in coronal holes in previous works, and as compared to the requirement of most theoretical models to accelerate the fast solar wind.

In Paper I, we showed that the instrumental stray light explains most of the line width decrease as a function of the altitude. We did not stress this aspect in this paper, as this result is not at all modified by the revision of the non-thermal velocity. The conclusion that there is no direct evidence for any substantial damping of the Alfvén waves above 200 ", as often stated, therefore remains true.

In fact, one can consider that the new results ascertain the fact that the Alfvén waves may not be damped. Initially, the method presented here was intended for providing an upper boundary for the non-thermal velocity, to analyse the preferential heating issue in what is the least favorable case, that is, undamped Alfvén waves. In Paper I, there remained some heating of the ions once the non-thermal velocity was removed. This implied that the heating could be more important, and that any damping of the Alfvén waves could be masked by an increase of the temperature. This can still be the case, but the rather flat temperature profiles that are obtained for $\mathrm{Mg}$ x especially, by removing the new value of $\xi$ (Fig. 4, left panel), may be the true ones. Indeed, the lack of heating of the high- $q / m$ ions may not be coincidental, since it is consistent with the numerical simulations mentionned above (Sect. 3.2). Hence, the red curve in Fig. 3 (i.e. undamped Alfvén waves) may also be the real non-thermal velocity, and not only an upper boundary.

The major difference with Paper I is, then, that we can no longer firmly conclude in favour of a preferential heating between $60^{\prime \prime}$ and any altitude above for ion species having low $q / m$ ratios. Except mainly for Fe $\mathrm{x}$, the increase of linewidth can be completely explained by an increase of non-thermal velocity with the altitude. Such a heating may exist anyhow, especially if the waves are in fact damped. But we have no direct evidence for that. Anyway, what is still true is that the derived ion temperatures are larger than the electron one usually measured in coronal holes. This is confirmed by the newly included Fe VIII, whose temperature is as large as the $\mathrm{Fe} \mathrm{x}$ one. Consequently, some process must have heated the ions preferentially to the electrons at altitudes lower than that where we observed. Work is currently under way to investigate this aspect with other SUMER and EIS/Hinode observations. 
Acknowledgements. The authors thank A. Zhukov for useful comments on the manuscript. The data analysed here were acquired during MEDOC Campaigns at IAS (http://www.medoc-ias.u-psud.fr). SOHO is a mission of international cooperation between ESA and NASA. The SUMER project is financially supported by DLR, CNES, NASA, and ESA PRODEX Programme (Swiss contribution). CHIANTI is a collaborative project involving the NRL (USA), RAL (UK), and the following Univerisities: College London (UK), Cambridge (UK), George Mason (USA), and Florence (Italy). We thank the anonymous referees for useful comments that improved the content of this article.

Topical Editor R. Forsyth thanks two anonymous referees for their help in evaluating this paper.

\section{References}

Banerjee, D., Teriaca, L., Doyle, J. G., and Wilhelm, K.: Broadening of Si VIII lines observed in the solar polar coronal holes, A\&A, 339, 208-214, 1998.

Bourouaine, S., Marsch, E., and Vocks, C.: On the Efficiency of Nonresonant Ion Heating by Coronal Alfvén Waves, ApJ, 684, L119-L122, doi:10.1086/592243, 2008a.

Bourouaine, S., Vocks, C., and Marsch, E.: Multi-Ion Kinetic Model for Coronal Loop, ApJ, 680, L77-L80, doi:10.1086/ 589741, 2008b.

Cranmer, S. R., Panasyuk, A. V., and Kohl, J. L.: Improved Constraints on the Preferential Heating and Acceleration of Oxygen Ions in the Extended Solar Corona, ApJ, 678, 1480-1497, doi: 10.1086/586890, 2008.

Curdt, W., Brekke, P., Feldman, U., Wilhelm, K., Dwivedi, B. N., Schühle, U., and Lemaire, P.: The SUMER spectral atlas of solardisk features, A\&A, 375, 591-613, 2001.

David, C., Gabriel, A. H., Bely-Dubau, F., Fludra, A., Lemaire, P., and Wilhelm, K.: Measurement of the electron temperature gradient in a solar coronal hole, A\&A, 336, L90-L94, 1998.

Del Zanna, G., Rozum, I., and Badnell, N. R.: Electron-impact excitation of Be-like Mg, A\&A, 487, 1203-1208, doi:10.1051/ 0004-6361:200809998, 2008.

Dere, K. P. and Mason, H. E.: Nonthermal velocities in the solar transition zone observed with the high-resolution telescope and spectrograph, Solar Phys., 144, 217-241, doi:10.1007/ BF00627590, 1993.

Dere, K. P., Landi, E., Mason, H. E., Monsignori Fossi, B. C., and Young, P. R.: CHIANTI - an atomic database for emission lines, A\&AS, 125, 149-173, doi:10.1051/aas:1997368, 1997.

Dolla, L. and Solomon, J.: Solar off-limb line widths: Alfvén waves, ion-cyclotron waves, and preferential heating, A\&A, 483, 271-283, doi:10.1051/0004-6361:20077903, 2008.

Dolla, L., Lemaire, P., Solomon, J., and Vial, J.-C.: A search for turbulent wave heating and acceleration signatures with SOHO/SUMER observations : Measurements of the widths of off-limb Iron lines, in: AIP Conf. Proc. 679: Solar Wind Ten, pp. 351-354, 2003.

Dolla, L., Solomon, J., and Lemaire, P.: A Search for Signatures of Preferential Heating by Ion Cyclotron Waves in the Low Corona, in: ESA SP-547: SOHO 13 Waves, Oscillations and Small-Scale Transients Events in the Solar Atmosphere: Joint View from SOHO and TRACE, p. 391, 2004.
Doschek, G. A., Warren, H. P., Laming, J. M., Mariska, J. T., Wilhelm, K., Lemaire, P., Schuehle, U., and Moran, T. G.: Electron Densities in the Solar Polar Coronal Holes from Density Sensitive Line Ratios of Si8 and Si10, ApJ, 482, L109-L112, doi: 10.1086/310668, 1997.

Ekberg, J. O. and Feldman, U.: New identifications of Mn VII and Fe VIII lines belonging to transitions of the type $3 p^{6} 4 p-3 p^{6} 4 d$ and $3 p^{5} 3 d^{2}-3 p^{6} 4 d$, ApJS, 595, 517-521, doi:10.1086/377300, 2003.

Feldman, U., Behring, W. E., Curdt, W., Schuehle, U., Wilhelm, K., Lemaire, P., and Moran, T. M.: A Coronal Spectrum in the 500-1610 Angstrom Wavelength Range Recorded at a Height of 21,000 Kilometers above the West Solar Limb by the SUMER Instrument on Solar and Heliospheric Observatory, ApJS, 113, 195-219, doi:10.1086/313048, 1997.

Habbal, S. R., Esser, R., and Arndt, M. B.: How reliable are coronal hole temperatures deduced from observations?, ApJ, 413, 435444, doi:10.1086/173011, 1993.

Harrison, R. A., Hood, A. W., and Pike, C. D.: Off-limb EUV line profiles and the search for wave activity in the low corona, A\&A, 392, 319-327, 2002.

Hassler, D. M., Rottman, G. J., Shoub, E. C., and Holzer, T. E.: Line broadening of Mg X 609 and $625 \AA$ coronal emission lines observed above the solar limb, ApJ, 348, L77-L80, doi:10.1086/ $185635,1990$.

Hollweg, J. V. and Isenberg, P. A.: Generation of the fast solar wind: A review with emphasis on the resonant cyclotron interaction, J. Geophy. Res. (Space Physics), 107, 1147, doi: 10.1029/2001JA000270, 2002.

Hu, Y. Q., Esser, R., and Habbal, S. R.: A four-fluid turbulencedriven solar wind model for preferential acceleration and heating of heavy ions, J. Geophys. Res., 105, 5093-5112, 2000.

Isenberg, P. A. and Vasquez, B. J.: Preferential Acceleration and Perpendicular Heating of Minor Ions in a Collisionless Coronal Hole, ApJ, 696, 591-600, doi:10.1088/0004-637X/696/1/591, 2009.

Landi, E. and Cranmer, S. R.: Ion Temperatures in the Low Solar Corona: Polar Coronal Holes at Solar Minimum, ApJ, 691, 794 805, doi:10.1088/0004-637X/691/1/794, 2009.

Landi, E., Del Zanna, G., Young, P. R., Dere, K. P., Mason, H. E., and Landini, M.: CHIANTI-An Atomic Database for Emission Lines. VII. New Data for X-Rays and Other Improvements, ApJS, 162, 261-280, doi:10.1086/498148, 2006.

Mierla, M., Schwenn, R., Teriaca, L., Stenborg, G., and Podlipnik, B.: Analysis of the Fe X and Fe XIV line width in the solar corona using LASCO-C1 spectral data, A\&A, 480, 509-514, doi:10.1051/0004-6361:20078329, 2008.

Moran, T. G.: Interpretation of coronal off-limb spectral line width measurements, A\&A, 374, L9-L11, doi:10.1051/0004-6361: 20010643, 2001.

Ofman, L., Davila, J. M., Nakariakov, V. M., and Viñas, A.-F.: High-frequency Alfvén waves in multi-ion coronal plasma: Observational implications, J. Geophys. Res. (Space Physics), 110, 9102, doi:10.1029/2004JA010969, 2005.

Parker, E. N.: Heating solar coronal holes, ApJ, 372, 719-727, doi: 10.1086/170015, 1991.

Schwenn, R. and Marsch, E.: Physics of the Inner Heliosphere II, Particules, Waves and Turbulence, Springer-Verlag, 1991.

Tomczyk, S., McIntosh, S. W., Keil, S. L., Judge, P. G., Schad, 
T., Seeley, D. H., and Edmondson, J.: Alfvén Waves in the Solar Corona, Science, 317, 1192-1196, doi:10.1126/science. 1143304, 2007.

Tu, C.-Y. and Marsch, E.: Two-Fluid Model for Heating of the Solar Corona and Acceleration of the Solar Wind by High-Frequency Alfven Waves, Solar Phys., 171, 363-391, 1997.

Tu, C.-Y., Marsch, E., Wilhelm, K., and Curdt, W.: Ion Temperatures in a Solar Polar Coronal Hole Observed by SUMER on SOHO, ApJ, 503, 475, doi:10.1086/305982, 1998.

Van Doorsselaere, T., Brady, C. S., Verwichte, E., and Nakariakov, V. M.: Seismological demonstration of perpendicular density structuring in the solar corona, A\&A, 491, L9-L12, doi: 10.1051/0004-6361:200810659, 2008a.

Van Doorsselaere, T., Nakariakov, V. M., and Verwichte, E.: Detection of Waves in the Solar Corona: Kink or Alfvén?, ApJ, 676, L73-L75, doi:10.1086/587029, 2008b.

Vocks, C.: A Kinetic Model for Ions in the Solar Corona Including Wave-Particle Interactions and Coulomb Collisions, ApJ, 568, 1017-1029, doi:10.1086/338884, 2002.
Vocks, C. and Marsch, E.: Kinetic Results for Ions in the Solar Corona with Wave-Particle Interactions and Coulomb Collisions, ApJ, 568, 1030-1042, doi:10.1086/338885, 2002.

von Steiger, R., Geiss, J., Gloeckler, G., and Galvin, A. B.: Kinetic Properties of Heavy Ions in the Solar Wind From SWICS/Ulysses, Space Sci. Rev., 72, 71-76, doi:10.1007/ BF00768756, 1995.

Wilhelm, K., Curdt, W., Marsch, E., Schuhle, U., Lemaire, P., Gabriel, A., Vial, J.-C., Grewing, M., Huber, M. C. E., Jordan, S. D., Poland, A. I., Thomas, R. J., Kuhne, M., Timothy, J. G., Hassler, D. M., and Siegmund, O. H. W.: SUMER - Solar U1traviolet Measurements of Emitted Radiation, Solar Phys., 162, 189-231, 1995.

Wilhelm, K., Marsch, E., Dwivedi, B. N., Hassler, D. M., Lemaire, P., Gabriel, A. H., and Huber, M. C. E.: The Solar Corona above Polar Coronal Holes as Seen by SUMER on SOHO, ApJ, 500, 1023-1038, doi:10.1086/305756, 1998.

Wilhelm, K., Fludra, A., Teriaca, L., Harrison, R. A., Dwivedi, B. N., and Pike, C. D.: The widths of vacuum-ultraviolet spectral lines in the equatorial solar corona observed with CDS and SUMER, A\&A, 435, 733-741, 2005. 